\title{
Sertoli-Leydig cell tumour: a rare cause of androgenic alopecia in post-menopausal women
}

\author{
S Siyambalapitiya ${ }^{1}$, J Fernado², R N G Rajapakse ${ }^{1}$ \\ Sri Lanka Journal of Diabetes, Endocrinology and Metabolism 2012; 2: 111-113
}

(Index words: Sertoli-Leydig cell tumour, ovarian tumour, postmenopausal women, hyperandrogenism, alopecia)

\begin{abstract}
Objective: Sertoli-Leydig cell tumour (SLCT) is a rare ovarian tumour often detected in young females between the ages of 20 and 30 years who usually presents with virilising features. We are describing an unusual case of Sertoli-Leydig cell tumour in a 57 year old postmenopausal woman who presented with progressive male pattern hair loss. She had high testosterone levels with poor response to medical therapy and underwent bilateral oophorectomy. Histology showed a Sertoli-Leydig cell tumour in the right ovary with benign characteristics and surgery resulted in an almost complete cure for the patient.
\end{abstract}

\section{Introduction}

Sertoli-Leydig cell tumour (SLCT) is a rare ovarian tumour belonging to the group of sex-cord stromal tumours. These neoplasms account for less than $0.5 \%$ of all ovarian tumours and are more often detected in young females between the ages of 20 and 30 years who usually become virilised (1) and very rarely in postmenopausal women. SLCTs are characterized by the presence of testicular structures that produce androgens. Most tumours are unilateral and confined to the ovaries. With this background, we describe an unusual case of SertoliLeydig cell tumour in a postmenopausal woman who presented with progressive hair loss. Despite, similar cases of SLCT of ovary in post-menopausal women have been reported (2-9), we believe this to be a useful addition to the literature.

\section{Case report}

A 57-year old married woman was referred to the endocrine clinic from the out patient department with progressive male pattern hair loss for 2 years' duration. She was married with 2 children. She had breast fed uneventfully and she had experienced the menopause at the age of 52. She noticed progressive hair loss especially from the forehead and the central area of the scalp over the last 2 years. She never had a similar problem, any other chronic medical illness or any skin disorder in the past.

On examination, she had male pattern hair loss (Figure 1) and persistently elevated blood pressure. Her glucose levels were repeatedly in the diabetic range. There was no hirsutism or clitoromegaly. CT scan of the abdomen showed normal ovaries and adrenals. The test results indicated excessive androgenic activity in the form of elevated serum testosterone levels (3.39 ng/mL; normal, 0.2-1.2 ng/mL) with normal dehydroepiandrosterone sulfate (DHEAS) levels.

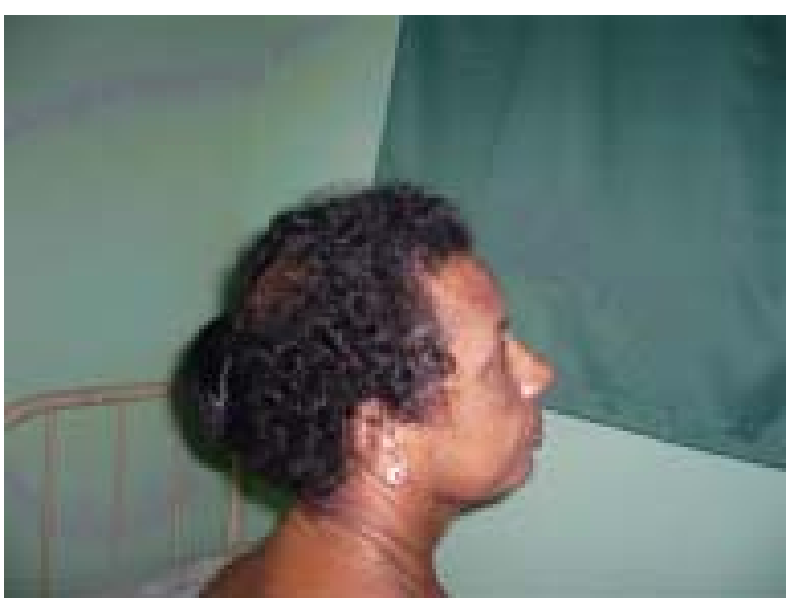

Figure 1. Figure shows the hair loss from forehead and central region of scalp.

Diabetes due to insulin resistance, hyperandrogenism and normal ovaries and adrenals in abdominal CT were in favour of ovarian hyperthecosis and we put her on the anti-androgens; spironolactone and finasteride. Metformin, glibenclamide, enalapril and atorvastatin were prescribed for treatment of diabetes and hypertension. Her blood glucose and the blood pressure were normalized with the medication. However, she had minimal response

${ }^{1}$ North Colombo Teaching Hospital, Ragama, Sri Lanka, ${ }^{2}$ National Hospital of Sri Lanka, Colombo. 
to anti-adrogen therapy and continued to loose hair. Therefore, we offered her the surgical treatment option, bilateral oophorectomy.

During surgery, the right ovary measuring $5 \times 1.8 \times 2 \mathrm{~cm}$ and the left ovary measuring $3 \times 2 \times 1 \mathrm{~cm}$ were removed. Cut surface of the right ovary showed a greyish white, well circumscribed nodule (measuring $2.5 \times 1.6 \times 1 \mathrm{~cm}$ ) towards the outer surface of the right ovary (Figure 2). Sections of this ovary showed well circumscribed tumour composed of sheets and clusters of cells with abundant eosinophillic cytoplasm. Some cells exhibited intracytoplasmic lipids with lipofuchsin pigment and there were clusters of nuclear rich areas separated by nuclear free areas. There were no nuclear atypia or increased mitotic activity. These findings were compatible with a benign Sertoli-Leydig cell tumour of the ovary (Figure 3). Cut surface of the left ovary showed a unilocular cyst (measuring $1 \mathrm{~cm}$ ) within the ovarian tissue, which was compatible with a seruous cystadenoma. However, rest of the left ovary appeared unremarkable.

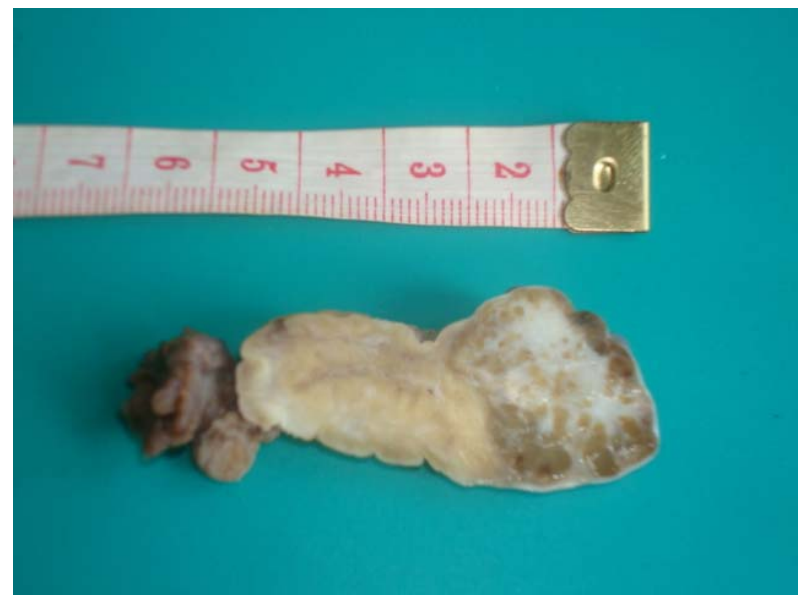

Figure 2. Cut surface of right ovary shows the greyish white, well demarcated Sertoli-Leydig cell tumour.

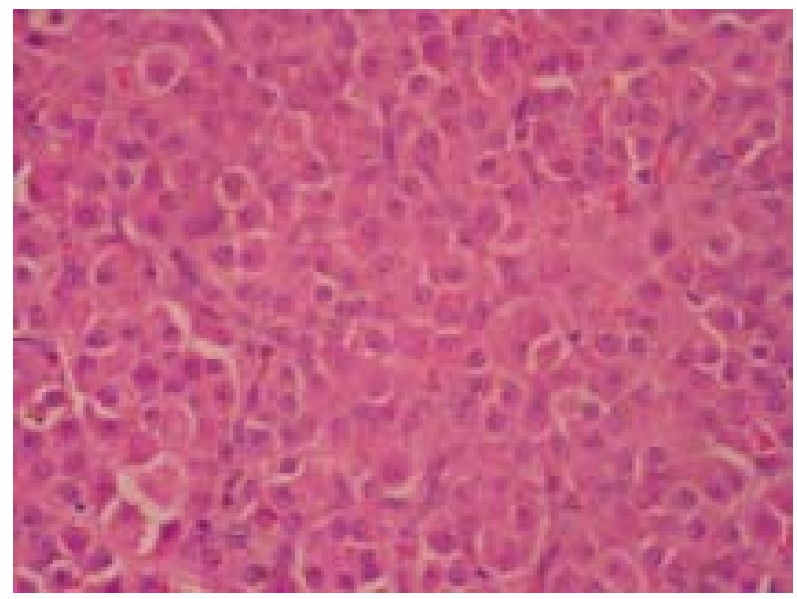

Figure 3. Sections of the right ovarian tumour shows sheets and clusters of cells with abundant eosinophillic cytoplasm, which is compatible with a Sertoli-Leydig cell tumour.
Four weeks after the operation, we reviewed the patient with the histo-pathological report and repeated testosterone results. The testosterone level had decreased to normal levels. The patient's symptoms have improved substantially and she had no further hair loss after the surgery. We discussed the results with the patient and decided to follow up her without subjecting to any further treatment.

\section{Discussion}

The majority of patients of SLCT are being detected during the second and third decades of life and SLCT at postmenopausal age is extremely rare. Very rarely, the SLCT can be associated with DHEAS secreting adrenal adenomas (7). Most important prognostic factors in these tumours are their stage and degree of differentiation. A histopathological review done by Young and Scully in 1985 (1), has shown that all well-differentiated tumours were benign, whereas $11 \%$ of tumours with intermediate differentiation, $59 \%$ of tumours with poor differentiation, and $19 \%$ of those with heterologous elements were malignant. In another study, patients who had intermediate or poorly differentiated SLCT, a survival rate of $92 \%$ was noted at both 5 and 10 years (10).

Most of these tumours are unilateral and diagnosed early; hence a conservative surgery is the only treatment that is needed. Adjuvant chemotherapy is considered for patients who have poor prognostic factors (10). Our patient had a well-differentiated tumour with benign characteristics and surgery alone was adequate for her as treatment.

In conclusion, SLCT is a rare ovarian sex-cord tumour that usually occurs unilaterally in young females and extremely rare in post-menopausal women. It is a rare cause of hyperandrogenism that needs to be considered in patients with virilising symptoms and signs.

\section{References}

1. Young RH, Scully RE. Ovarian Sertoli-Leydig cell tumors. A clinicopathological analysis of 207 cases. The American Journal of Surg Pathology 1985; 9: 543-69.

2. Mango D, Scirpa P, Liberati M, Menini E, Fabiano A, Mancuso S. Ovarian and peripheral steroid hormones in a case of Sertoli-Leydig cell tumor. Journal of Endocrinological Investigation 1988; 11: 521-5.

3. Hansen TP, Sorensen B. Sertoli-Leydig cell tumour of the ovary - a rare cause of virilization after menopause. Apmis 1993; 101: 663-6.

4. Gheorghisan-Galateanu A, Fica S, Terzea DC, Caragheorgheopol A, Horhoianu V. Sertoli-Leydig cell tumor - a rare androgen secreting ovarian tumor in postmenopausal women: a case report and review of literature. Journal of Cellular and Molecular Medicine 2003; 7: 461-71. 
5. Genton C. A Sertoli-Leydig cell tumor as an incidental finding in a 72 year old patient. Zentralblatt fur Gynakologie 1978; 100: $154-6$.

6. Dahlgren E, Gull B, Willen R, Sundler F, Rosen T, Jansson PA. Sertoli-Leydig cell tumour in a postmenopausal woman showing all facets of the insulin resistance syndrome (IRS). Upsala Journal of Medical Sciences 2005; 110: 233-6.

7. Herrera JD, Davidson JA, Mestman JH. Hyperandrogenism due to a testosterone-secreting Sertoli-Leydig cell tumor associated with a dehydroepiandrosterone sulfate-secreting adrenal adenoma in a postmenopausal woman: case presentation and review of literature. Endocrine Practice 2009; 15(2): 149-52.
8. Yetkin DO, Demirsoy ET, Kadioglu P. Pure Leydig cell tumour of the ovary in a post-menopausal patient with severe hyperandrogenism and erythrocytosis. Gynecological Endocrinology 2011; 27(4): 237-40.

9. Marcelino M, Nobre E, Conceição J, Lopes L, Vilar H, França Martins M, Carvalho A, André S, Horta A, De Castro JJ. A rare case of hyperandrogenism: bilateral Leydig cell tumor of the ovary. Acta Medica Portusuea 2010; 23(1): 113-8.

10. Zaloudek C, Norris HJ. Sertoli-Leydig tumors of the ovary. A clinicopathologic study of 64 intermediate and poorly differentiated neoplasms. The American Journal of Surgical Pathology 1984; 8: 405-18. 